\title{
An apparatus for generation and quantitative measurement of homogeneous isotropic turbulence in $\mathrm{He}$ ii
}

Brian Mastracci, and Wei Guo

Citation: Review of Scientific Instruments 89, 015107 (2018); doi: 10.1063/1.4997735

View online: https://doi.org/10.1063/1.4997735

View Table of Contents: http://aip.scitation.org/toc/rsi/89/1

Published by the American Institute of Physics

\section{Articles you may be interested in}

A local sensor for joint temperature and velocity measurements in turbulent flows

Review of Scientific Instruments 89, 015005 (2018); 10.1063/1.4989430

A new ultrasonic transducer sample cell for in situ small-angle scattering experiments

Review of Scientific Instruments 89, 015111 (2018); 10.1063/1.5021370

Improving the magnetic field homogeneity by varying magnetic field structure in a geophone

Review of Scientific Instruments 89, 015008 (2018); 10.1063/1.5005847

Measurement and control from frequency to phase based on virtual signal reconstruction

Review of Scientific Instruments 89, 015112 (2018); 10.1063/1.5019288

Development of a multistage compliant mechanism with new boundary constraint

Review of Scientific Instruments 89, 015009 (2018); 10.1063/1.5007890

Note: Contrast enhancement and artifact suppression in computed tomography using sinogram normalization Review of Scientific Instruments 89, 016101 (2018); 10.1063/1.5004061

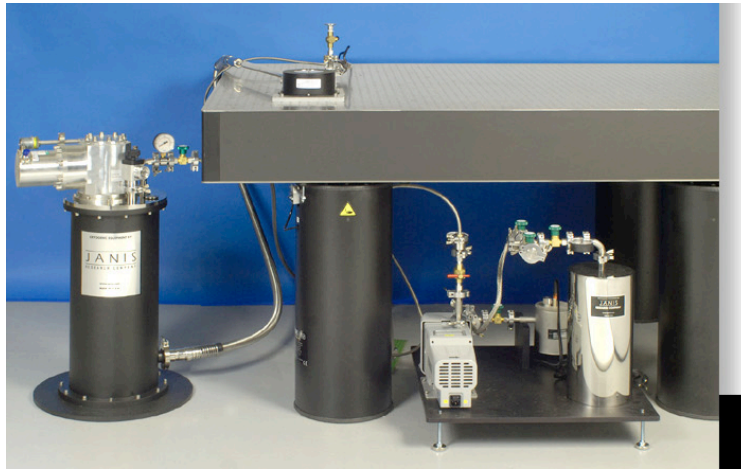

sales@janis.com

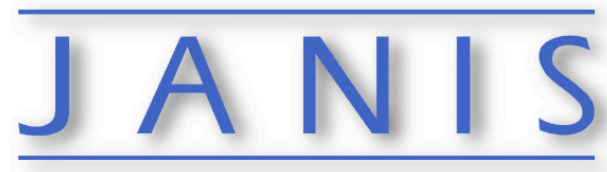

Rising LHe costs? Janis has a solution. Janis' Recirculating Cryocooler eliminates the use of Liquid Helium for "wet" cryogenic systems. 


\title{
An apparatus for generation and quantitative measurement of homogeneous isotropic turbulence in He II
}

\author{
Brian Mastracci ${ }^{1,2}$ and Wei Guo ${ }^{1,2, a)}$ \\ ${ }^{1}$ National High Magnetic Field Laboratory, 1800 E Paul Dirac Dr., Tallahassee, Florida 32310, USA \\ ${ }^{2}$ Department of Mechanical Engineering, Florida State University, 2525 Pottsdamer St., Tallahassee, \\ Florida 32310, USA
}

(Received 26 July 2017; accepted 31 December 2017; published online 19 January 2018)

\begin{abstract}
The superfluid phase of helium-4, known as He II, exhibits extremely small kinematic viscosity and may be a useful tool for economically producing and studying high Reynolds number turbulent flow. Such applications are not currently possible because a comprehensive understanding of the complex two-fluid behavior of He II is lacking. This situation could be remedied by a systematic investigation of simple, well controlled turbulence that can be directly compared with theoretical models. To this end, we have developed a new apparatus that combines flow visualization with second sound attenuation to study turbulence in the wake of a mesh grid towed through a He il filled channel. One of three mesh grids (mesh number $M=3,3.75$, or $5 \mathrm{~mm}$ ) can be pulled at speeds between 0.1 and $60 \mathrm{~cm} / \mathrm{s}$ through a cast acrylic flow channel which has a $16 \mathrm{~mm} \times 16 \mathrm{~mm}$ cross section and measures $330 \mathrm{~mm}$ long. The motion of solidified deuterium tracer particles, with diameter of the order $1 \mu \mathrm{m}$, in the resulting flow is captured by a high speed camera, and a particle tracking velocimetry algorithm resolves the Lagrangian particle trajectories through the turbulent flow field. A pair of oscillating superleak second sound transducers installed in the channel allows complementary measurement of vortex line density in the superfluid throughout the turbulent decay process. Success in early experiments demonstrates the effectiveness of both probes, and preliminary analysis of the data shows that both measurements strongly correlate with each other. Further investigations will provide comprehensive information that can be used to address open questions about turbulence in He II and move toward the application of this fluid to high Reynolds number fluid research. Published by AIP Publishing. https://doi.org/10.1063/1.4997735
\end{abstract}

\section{INTRODUCTION}

Cryogenic helium-4 has considerable potential in the future of fluids research due to a number of thermal and mechanical properties. ${ }^{1,2}$ In particular, the kinematic viscosity $v$ of liquefied ${ }^{4} \mathrm{He}$ can be lower than $10^{-8} \mathrm{~m}^{2} / \mathrm{s}$, smaller than the kinematic viscosity of any other fluid. ${ }^{3}$ Consequently, the Reynolds number, defined as $R e=D U / v$ (where $D$ represents a characteristic length and $U$ represents the flow velocity), for helium flows can be pushed to extremely high values within a compact laboratory apparatus. Using cryogenic ${ }^{4} \mathrm{He}$ as the working fluid, one could economically reproduce and study high Reynolds number turbulent flows such as those occurring in the Earth's atmosphere and oceans or around large transportation and defense vessels. $R e \sim 10^{7}$ have already been achieved in liquid helium pipe flow ${ }^{4}$ and von Kármán flow, and plans to achieve $R e \sim 10^{8}$ are in place. ${ }^{5}$ However, practical application of such high Reynolds number flow using helium is complicated by the fact that the smallest kinematic viscosities are exhibited by He II, the colder of two liquid phases that occur in ${ }^{4} \mathrm{He}$, and the turbulent behavior of this fluid is not sufficiently understood.

Below the $\lambda$-transition temperature $\left(T_{\lambda} \approx 2.17 \mathrm{~K}\right)$, ordinary liquid helium, or He $\mathrm{I}$, transitions to a superfluid phase known as $\mathrm{He}$ II. He II can be described as a fluid system

a) Author to whom correspondence should be addressed: wguo@ magnet.fsu.edu comprised of two fully miscible components: the superfluid component and the normal fluid component. ${ }^{6}$ At $T_{\lambda}$, the fluid system is comprised entirely of normal fluid, and its density $\rho_{n}$ relative to that of the bulk fluid $\rho$ is equal to unity. Conversely, the relative density of the superfluid, $\rho_{s} / \rho$, increases with decreasing temperature until the system consists entirely of superfluid in the zero temperature limit. This superfluid is inviscid and carries no entropy, and its vorticity is quantized in units of $\kappa=h / m_{4}$, where $h$ is Planck's constant and $m_{4}$ is the mass of a ${ }^{4} \mathrm{He}$ atom. The physical manifestation of turbulence in the superfluid is a random tangle of singly quantized, angstrom sized vortex lines. On the other hand, the normal fluid is a classical viscous fluid and behaves as such, except that its interaction with the quantized vortex tangle leads to a non-classical force referred to as mutual friction. ${ }^{7}$

This two-fluid system exhibits a number of interesting characteristics. For instance, in the presence of a heat source, the normal fluid component carries entropy away from the source while the superfluid moves toward it to conserve mass. ${ }^{6}$ This extremely efficient heat transfer mechanism, known as thermal counterflow, is unique to He II. Furthermore, in addition to ordinary sound in the sense of pressure waves in the bulk fluid, second sound, or entropy waves, can develop in He II when the relative densities of the two fluid components oscillate out of phase. ${ }^{6}$

Despite the complex two-fluid behavior and non-classical nature of the superfluid, experimental observations suggest that mechanically generated turbulent flow in $\mathrm{He}$ II behaves 
similarly to analogous turbulent flow in classical fluids. Classical Kolmogorov energy spectra and decay behavior have been observed for turbulence in the wake of a moving grid, ${ }^{8}$ between rotating propellers, ${ }^{5,9}$ and downstream of a stationary grid installed in a He II forced flow channel. ${ }^{10,11}$ This quasiclassical behavior has been attributed to coupling of the two fluid components due to mutual friction on length scales exceeding the average spacing between quantized vortices; ${ }^{12}$ turbulent eddies in the normal fluid component are matched by polarized bundles of vortices in the superfluid. ${ }^{13}$ However, not all research supports this picture of quasiclassical turbulence. Recent simulations suggest that eddies in the normal fluid and superfluid may not become locked together. ${ }^{14}$ Others point to non-classical features of turbulence in He II, such as temperature-dependent enhancement of intermittency, ${ }^{15}$ though a recent study of turbulent flow in the wake of a disc indicates that intermittency is not temperature dependent. ${ }^{16}$

Previous experimental methods do not provide enough evidence to resolve this confusing picture of quasiclasscal turbulence. A widely adopted tool for $\mathrm{He}$ II research is the measurement of second sound attenuation, which takes advantage of the scattering of second sound waves from quantized vortices to measure vortex line length per unit volume in the superfluid, but this method provides no information about the normal fluid component. More traditional probes such as pressure tubes monitor the mixed effects of both fluids, but due to their size they have limited spatial resolution. The invasive nature of such probes can introduce substantial turbulence that is difficult to separate from that generated intentionally, as can other factors such as the boundary layer established by flow past the container walls. To conclusively test the two fluid coupling concept and characterize the properties of quasiclassical turbulence, a systematic study of the behavior of both fluid components under simple and well controlled conditions is imperative.

A possible approach is the use of flow visualization to study quasiclassical turbulence in the wake of a grid pulled through a container of quiescent He II. Flow in the wake of a towed grid is generally accepted to be homogeneous isotropic turbulence (HIT), a simple form of turbulence that has received extensive theoretical and experimental attention in classical fluids research. ${ }^{17}$ Direct visualization offers high resolution and unobtrusive measurement of the entire twodimensional flow field in the bulk fluid. Visualization of grid turbulence has been successfully applied to $\mathrm{He} \mathrm{I},{ }^{18,19}$ the classical liquid phase, but applying the same to He II requires a number of non-trivial considerations. For example, new methods for introducing passive tracer particles into $\mathrm{He}$ II reduce unwanted heating and turbulence ${ }^{20}$ and incorporating second sound attenuation would provide a more comprehensive measurement of the fluid behavior. Additionally, the magnetic coupling between the grid and drive system used for $\mathrm{He}$ I grid turbulence ${ }^{18}$ is unlikely to provide careful control of the grid motion. Very recently, visualization of oscillating grid turbulence in $\mathrm{He}$ II has been reported, ${ }^{21}$ though the oscillating object described in this report can hardly be called a grid (the mesh occupies less than 50\% of the container cross section), and the ability to measure second sound attenuation was not mentioned (though it would not be of much use in the sustained oscillatory flow). While this device may perfectly suit the authors' research agenda, the need for comprehensive measurement of simple and well controlled turbulence is not satisfied. Considerable forethought and careful design are required to produce turbulence with the prescribed characteristics, and the flow generation and measurement mechanisms must work together to access information about the behavior of both fluid components.

This paper describes the design and successful operation of an apparatus that combines direct flow visualization with second sound attenuation to probe towed grid turbulence in He II. Section II describes the careful design of the system that produces HIT for which all constituents of the Reynolds number (length, velocity, and viscosity) can be systematically changed through control of the grid size, towing speed, and fluid temperature. Procedures for visualizing the bulk fluid velocity field using particle tracking velocimetry (PTV), as well as measuring the time-dependent vortex line density in the superfluid using second sound attenuation, as the turbulence decays are also discussed. Preliminary visualization results for He II grid turbulence are shown in Sec. III, together with vortex line density measurements that were obtained using the same apparatus. Both observations correlate strongly with each other, indicating the capability of this device to make comprehensive measurements of $\mathrm{He}$ II grid turbulence that were not previously possible. Two avenues for research are now available: first, a large number of turbulent flow statistics are derived from the velocity field, which is easily obtained through the visualization experiment; additionally, the behavior of the bulk fluid observed by visualization can be compared with the behavior of the superfluid component alone, observed using second sound attenuation, and the similarities and differences will reveal the degree to which the two fluid components are coupled. Section IV concludes by describing some of these applications in detail.

\section{EXPERIMENTAL DESIGN AND METHODS}

The apparatus for generating and visualizing grid turbulence consists of a vertical flow channel, grid and pulling system, and instrumentation and fits inside a standard liquid helium cryostat with optical access. These components are illustrated in Fig. 1. The cryostat consists of a cylindrical helium reservoir surrounded by several layers of low emissivity super insulation, vacuum, and liquid nitrogen shielding, which thermally isolate the reservoir from atmospheric conditions. Three sets of 2 in. fused silica viewports, spaced $90^{\circ}$ from each other, allow optical access for the visualization experiment. Evaporative cooling of the helium reservoir by a powerful vacuum system with base pressure less than 1 Torr, together with an electronic pressure control system, stabilizes the fluid temperature anywhere between $T_{\lambda}$ and about $1.2 \mathrm{~K}$.

Turbulence is generated and contained inside a cast acrylic channel, shown in additional detail in Fig. 2(a), which is held immersed in liquid helium inside the cryostat such that its geometric center is aligned with the cryostat viewports. Since the channel is immersed, the fluid inside is in thermal equilibrium with its surroundings, eliminating any heat leak that 


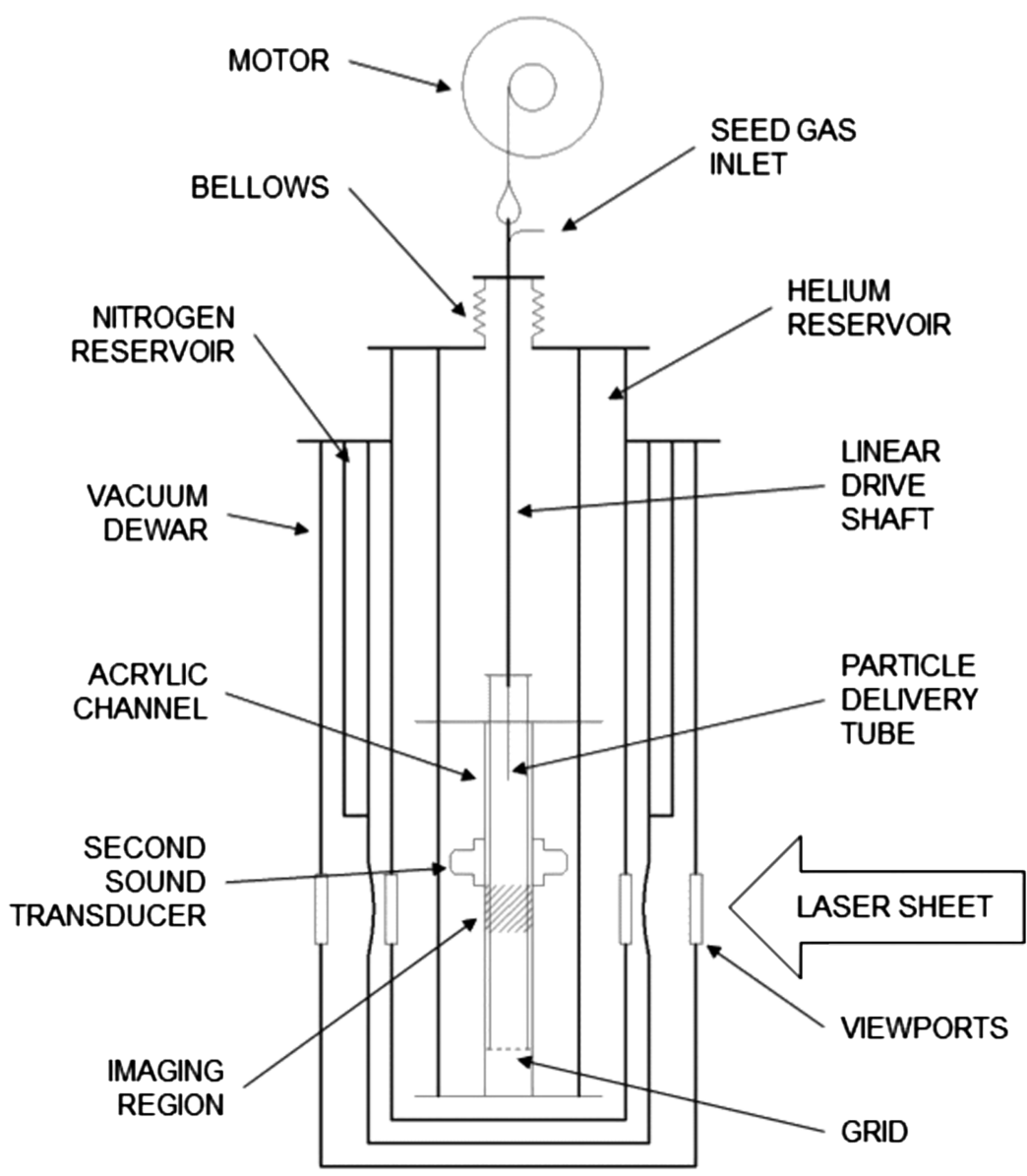

FIG. 1. Simple illustration of the overall experimental setup showing the cryostat, channel, and grid and pulling system (not to scale). might induce a thermal counterflow that interferes with the grid turbulence.

Acrylic is not a typical material for cryogenic service, but it satisfies a number of key requirements for the experiment: in the imaging region, the channel needs to be transparent; the interior walls need to be as smooth and uniform as possible, to prevent interference with the grid motion and introduction of unwanted turbulent flow structures; and, diagnostic instruments such as second sound transducers and temperature sensors need to be incorporated with the channel. Since acrylic is transparent and easy to machine, it is relatively easy to make an optical access channel with uniform walls without sacrificing mounting provisions for the instrumentation.

The channel measures $33 \mathrm{~cm}$ tall with cross section $1.6 \mathrm{~cm}$ square and is made from four pieces of cast acrylic which fit together as shown in Fig. 2(b). The edges of all four pieces were precisely machined to ensure the final assembly has a uniform square cross section along the entire length, and they were joined by chemically melting the acrylic with a commercially available solvent welding compound. ${ }^{22} \mathrm{~A}$ thinner piece is selected for the front wall (through which the camera looks) to prevent optical distortion in the image data. On the outside of the thicker back and side walls, several threaded hole patterns facilitate attachment of the second sound transducers (discussed in Sec. II B), temperature sensors, and a resistive heater.

The heater is sealed to the bottom end of the channel using indium wire and is included so that the facility can also be used for thermal counterflow experiments. It consists of four $100 \Omega$ surface mount resistors arranged in series across the bottom of the channel, as shown in Fig. 2(c). About 80\% of the bottom area is occupied by the resistors, ensuring uniform heating across the channel cross section.

Cernox ${ }^{\circledR}$ temperature sensors ${ }^{23}$ are placed in the back wall of the channel, $2.5 \mathrm{~cm}$ from the top and $2.5 \mathrm{~cm}$ from the bottom. The sensors are designed to measure the fluid temperature inside the channel without interrupting the smoothness of the interior walls. This was accomplished by encapsulating the sensors in epoxy. ${ }^{24}$ As a result, the sensing surface and epoxy form a smooth surface as shown in Fig. 2(d), and this surface is held flush with the interior channel wall by a carefully machined mounting flange.

Central to the grid turbulence facility is the mesh grid itself and the system that pulls it through the channel. In keeping with the overall objective to produce well-controlled HIT, particular attention has been given to the grid design. Oscillating grid experiments in water have revealed that if the grid solidity (the ratio of the solid surface area of the grid to the open area) exceeds about $40 \%$ and if the mesh is not symmetric with respect to the container walls, the resulting turbulence will be obstructed by large-scale, inhomogeneous secondary flow that does not behave in the manner predicted for HIT. ${ }^{25}$ More recent reports on experiments of the same kind stress that the size of any structure supporting the grid should not exceed that of the bars that form the grid mesh. ${ }^{26}$ Though the grid motion and working fluid will differ in this experiment, these guidelines 


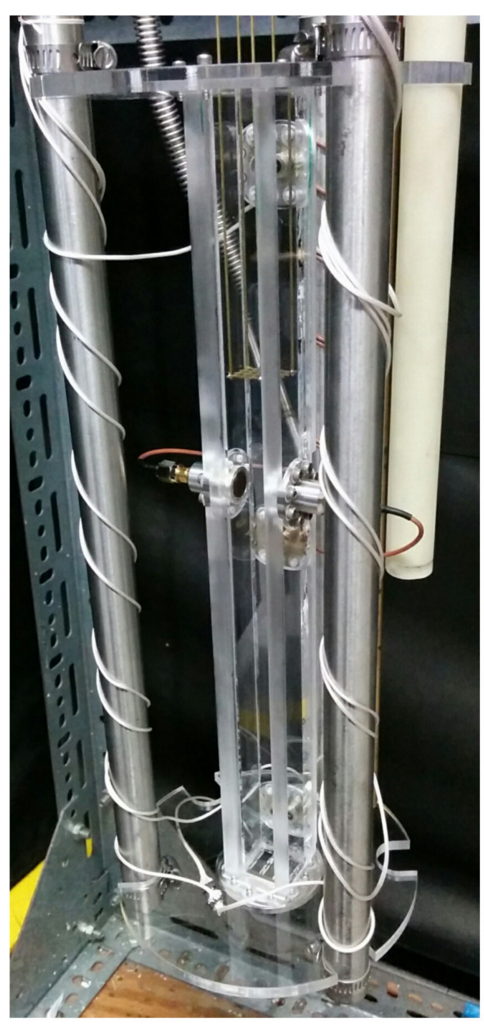

(a)

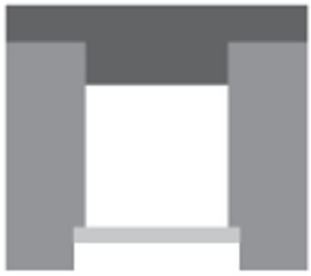

(b)

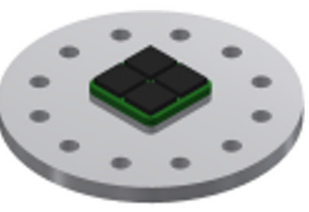

(c)

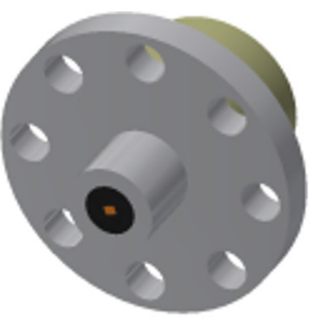

(d)
FIG. 2. (a) Cast acrylic flow channel developed for the study of grid turbulence in He II using both particle tracking velocimetry and second sound attenuation; (b) view of the channel along its axis, showing how the four pieces of acrylic come together; (c) resistive heater assembly that acts as the bottom wall; (d) encapsulated Cernox temperature sensors, mounted in a flange so that the sensing surface is flush with the interior channel wall.

for producing HIT are considered a good starting point. As an added requirement, since the large length-to-width ratio of the channel makes contact between the grid and walls inevitable, the influence of this contact on the resulting flow should be minimized.

Three grids with solidity $40 \%$ and different mesh sizes, $M=3,3.75$ and $5 \mathrm{~mm}$, were designed as shown in Fig. 3 and cut from brass sheet. The sheet stock thickness $(0.025$, 0.032 , and $0.040 \mathrm{in}$., respectively) was selected so that the bars have square cross section. In the corners, which represent the points farthest from the imaging region, the mesh pattern is interrupted to provide an attachment point for four $1 \mathrm{~mm}$ brass wires that connect the grid to the pulling system. The corners also extend slightly (about $0.4 \mathrm{~mm}$ ) beyond the edges of the mesh so that any contact between the grid and channel occurs at the corners, minimizing the contact area and keeping any influence on the turbulent flow as far from the imaging region as possible.
A 0.5 in. stainless steel tube serves as the main linear drive shaft, linking the four grid support wires just outside the channel to a cable outside the cryostat. An edge welded metal bellows, designed and manufactured ${ }^{27}$ specifically for this experiment, seals the shaft to the cryostat while it traverses the full $30 \mathrm{~cm}$ stroke (see Figs. 1 and 4). This method of sealing the linear drive shaft is preferred because it isolates the cold components of the system from atmospheric conditions throughout the entire range of motion and maintains direct mechanical connection between the motor and grid. Three polytetrafluoroethylene bearings, designed to minimize frictional heating by minimizing contact area and bearing stress at cryogenic temperatures, align the shaft inside the cryostat. A $3 \mathrm{~mm}$ cable is tied to the top of the shaft and routed through a series of pulleys, shown in Fig. 4, to a $32 \mathrm{~mm}$ drum attached to an electric stepper motor. This system has successfully pulled the grid at a number of different velocities, the smallest of which was $0.1 \mathrm{~cm} / \mathrm{s}$ and the largest $60 \mathrm{~cm} / \mathrm{s}$, and the desired velocity is maintained for at least the middle third of the stroke.

\section{A. Visualization experiment}

Before pulling the grid for the visualization experiment, the fluid must be seeded with tracer particles. These are generated by introducing a small amount of deuterium gas to He II through a $0.25 \mathrm{in}$. stainless steel tube, which is aligned with the channel axis and opens just above the imaging region. Since the linear drive shaft is already located above and concentric with the channel, the tracer particle delivery tube is placed inside of the shaft (see Fig. 1). This configuration allows tracer particles to be delivered to the optimal location, but the delivery tube does not interfere with the grid since the two move together.

A partial pressure blending system, shown in Fig. 5, is used to prepare the tracer particle seeding gas. Previous work has shown that a combination of low condensible gas partial pressure and low flow rate produces solidified tracer particles with size less than one micron in He II. $^{20}$ Therefore, a small quantity of deuterium is added to the evacuated mixture cylinder, which is then charged to $10 \mathrm{psig}$ with helium gas. The mixture is supplied at a regulated pressure of $600 \mathrm{mbar}$ and enters the delivery tube through a metering valve (flow coefficient $C_{v}<0.003$ ). The resulting particle size can be determined by measuring the terminal velocity $v_{\text {slip }}$ of the particles in quiescent $\mathrm{He}$ II ${ }^{28,29}$ which is a function of the particle size $d_{p}$ and is obtained by balancing the Stokes drag force acting on the particle $^{30}$ with its weight in liquid helium,

$$
v_{s l i p}=\frac{g\left(\rho_{f}-\rho_{p}\right) d_{p}}{18 \mu},
$$

where $\rho_{f}$ and $\mu$ are the density and dynamic viscosity, respectively, of the fluid, $\rho_{p}$ is the density of the particle, and $g$ is the
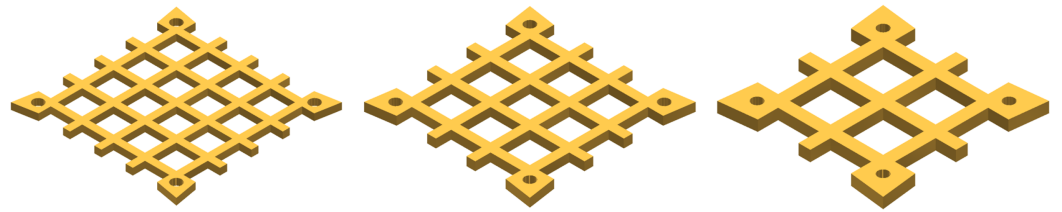

FIG. 3. Three mesh grids with mesh sizes, from left to right, of $M=3,3.75$, and $5 \mathrm{~mm}$ have been produced for the experiment. The corresponding bar width is 0.027 , 0.033 , and 0.044 in., respectively, and the grid thickness is $0.025,0.032$, and $0.040 \mathrm{in}$., respectively. 


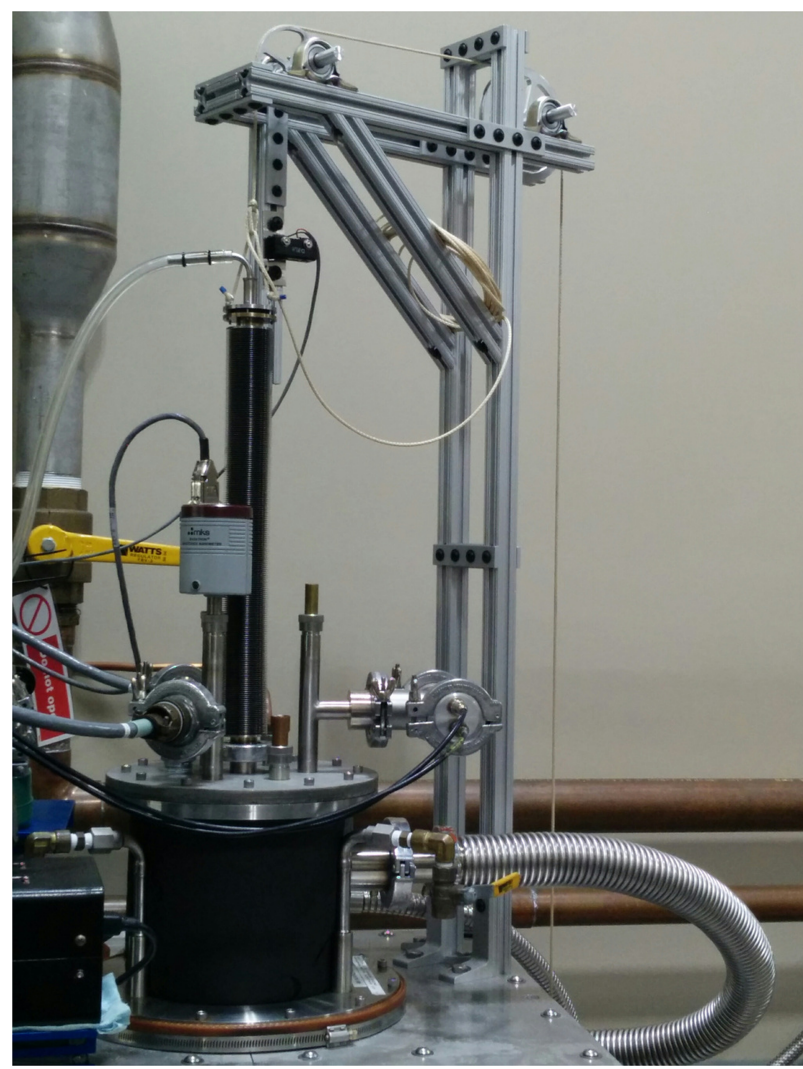

FIG. 4. Photograph of the top of the cryostat, showing the bellows and a portion of the pulling cable system.

acceleration due to gravity. Equation (1) can be rearranged to give the particle size as a function of the measured terminal velocity,

$$
d_{p}=\sqrt{\frac{18 \mu v_{\text {slip }}}{g\left(\rho_{f}-\rho_{p}\right)}} .
$$

Using this method, the slip velocity has been measured as $-0.42 \pm 0.25 \mathrm{~mm} / \mathrm{s}$, and the corresponding particle size, shown in Fig. 6, has been calculated as $4.6 \pm 1.3 \mu \mathrm{m}$. This essentially sets the resolution for the visualization experiment, as the particle size represents the smallest length scale that can be resolved. ${ }^{31}$ Since the inter-vortex spacing will be of the order $10 \mu \mathrm{m}$ or greater in most cases, these particles are sufficient for probing all length scales of interest (i.e., those exceeding the inter-vortex spacing, for which the two fluid components exhibit quasiclassical behavior).

A $473 \mathrm{~nm}$ continuous wave diode pumped solid state laser ${ }^{32}$ provides illumination for the tracer particles. In a typical experiment, the output power is adjusted to $400 \mathrm{~mW}$, and the beam shape is manipulated into a sheet measuring about $20 \mathrm{~mm}$ tall and $100 \mu \mathrm{m}$ thick where it passes through the cryostat. Light scattered by particles in the illuminated plane is collected by a high-speed CMOS camera ${ }^{33}$ with the image acquisition rate set to $100 / \mathrm{s}$, and the image resolution is 1280 pixels wide by 720 pixels high. A macro lens with adjustable focus maps the imaging plane, the width of which matches the width of the channel, onto the camera sensor. To extract particle tracks from the recorded sequence of images, a feature point tracking algorithm capable of resolving the particle location with sub-pixel resolution ${ }^{34}$ has been adapted to the experiment. Since position and time information are known for the particles in each track, the entire two-dimensional velocity field can be resolved, and the turbulent velocity statistics can be computed and compared with other classical and quasiclassical experimental results and theoretical predictions.

\section{B. Second sound experiment}

Determination of vortex line density by measuring second sound attenuation is based on the generation and pickup of second sound waves by oscillating superleak transducers (OST). These devices, commonly used for He II research,

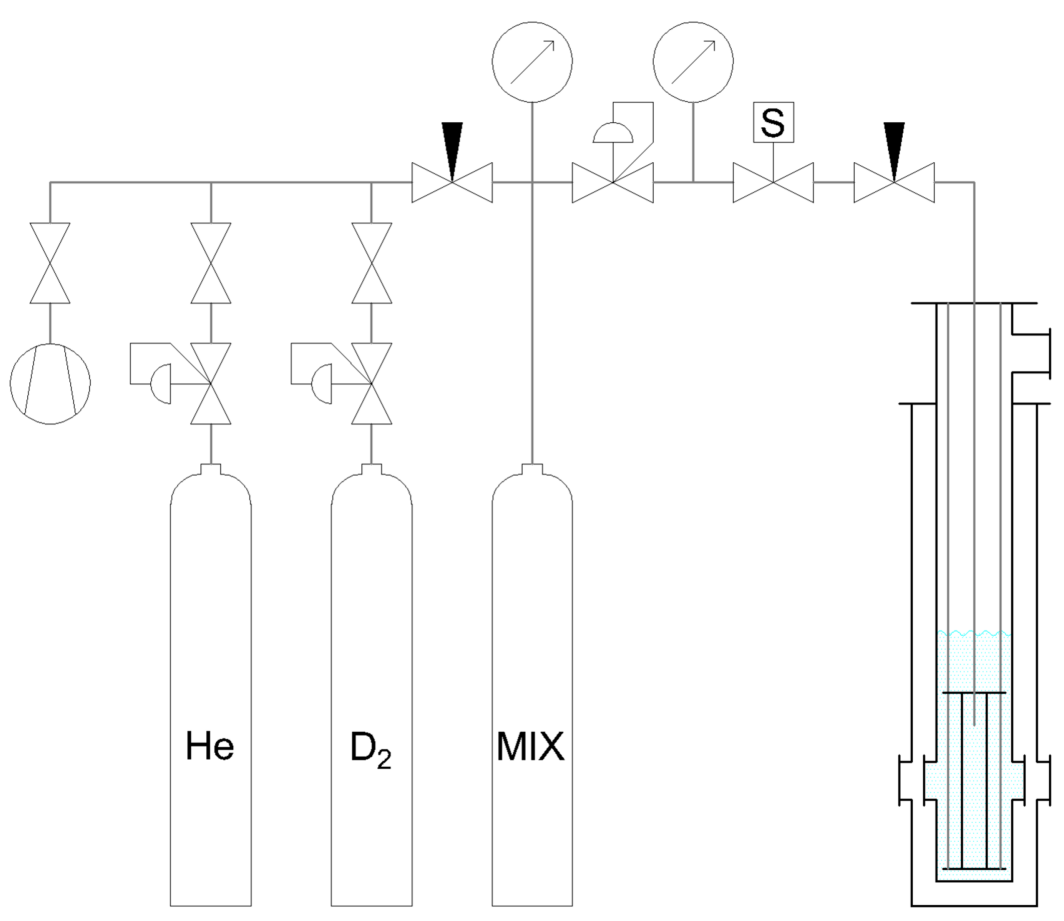

FIG. 5. Partial pressure blending system used to mix deuterium and helium gas for generating tracer particles and introducing them directly into $\mathrm{He}$ II inside the flow channel. The mixture ratio, injection time, and injection flow rate can be controlled by the gas handling system. 


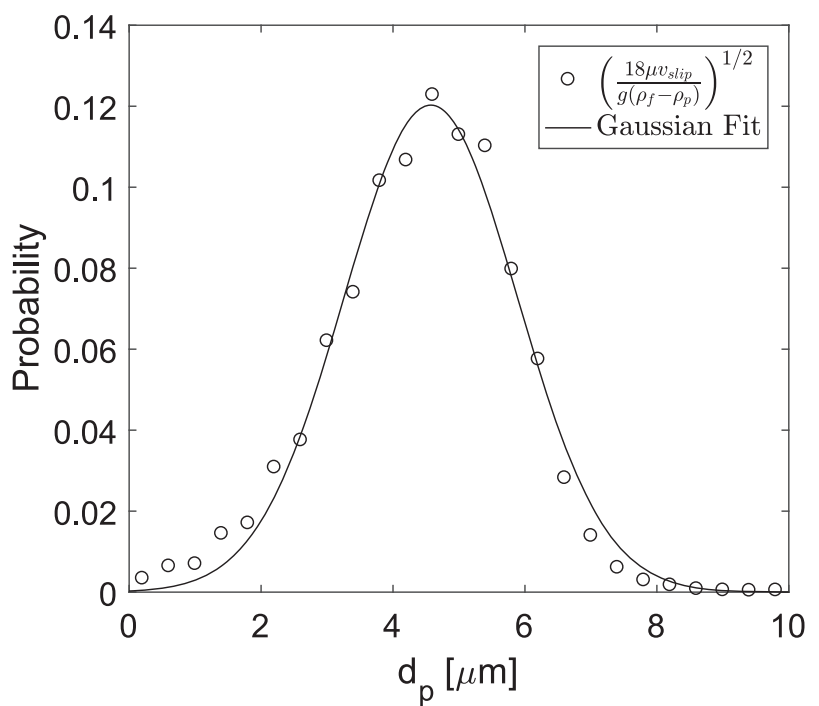

FIG. 6. Probability density function for solidified tracer particle size. The particle size, calculated from a terminal velocity measurement in quiescent He II, is $4.6 \pm 1.3 \mu \mathrm{m}$.

are essentially parallel plate capacitors with one fixed plate and one flexible plate. ${ }^{35} \mathrm{~A}$ thin porous membrane with an evaporated gold layer on one side, which serves as the dielectric and

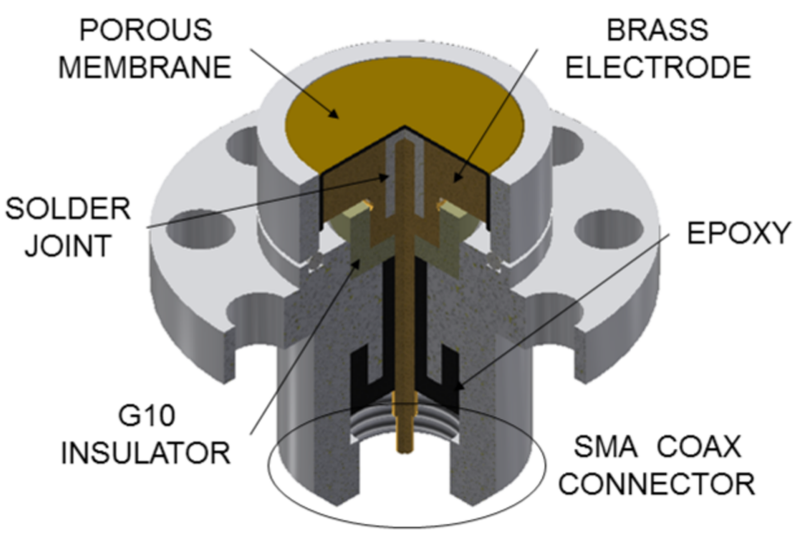

FIG. 7. Cross section of the custom designed second sound transducers used for the grid turbulence apparatus. For clarity, the polycarbonate membrane thickness is exaggerated, and the cut surface is shown in black.

flexible plate, is stretched over a rigid conductor, which serves as the fixed plate. Applying an alternating current to the transducer causes the membrane to vibrate. If it is exposed to He II, only the normal fluid component transmits the vibrations since the superfluid is inviscid and can slip through the pores. As a result, oscillations of the component densities $\rho_{n}$ and $\rho_{s}$, or second sound waves, develop in the fluid. Similarly, a second

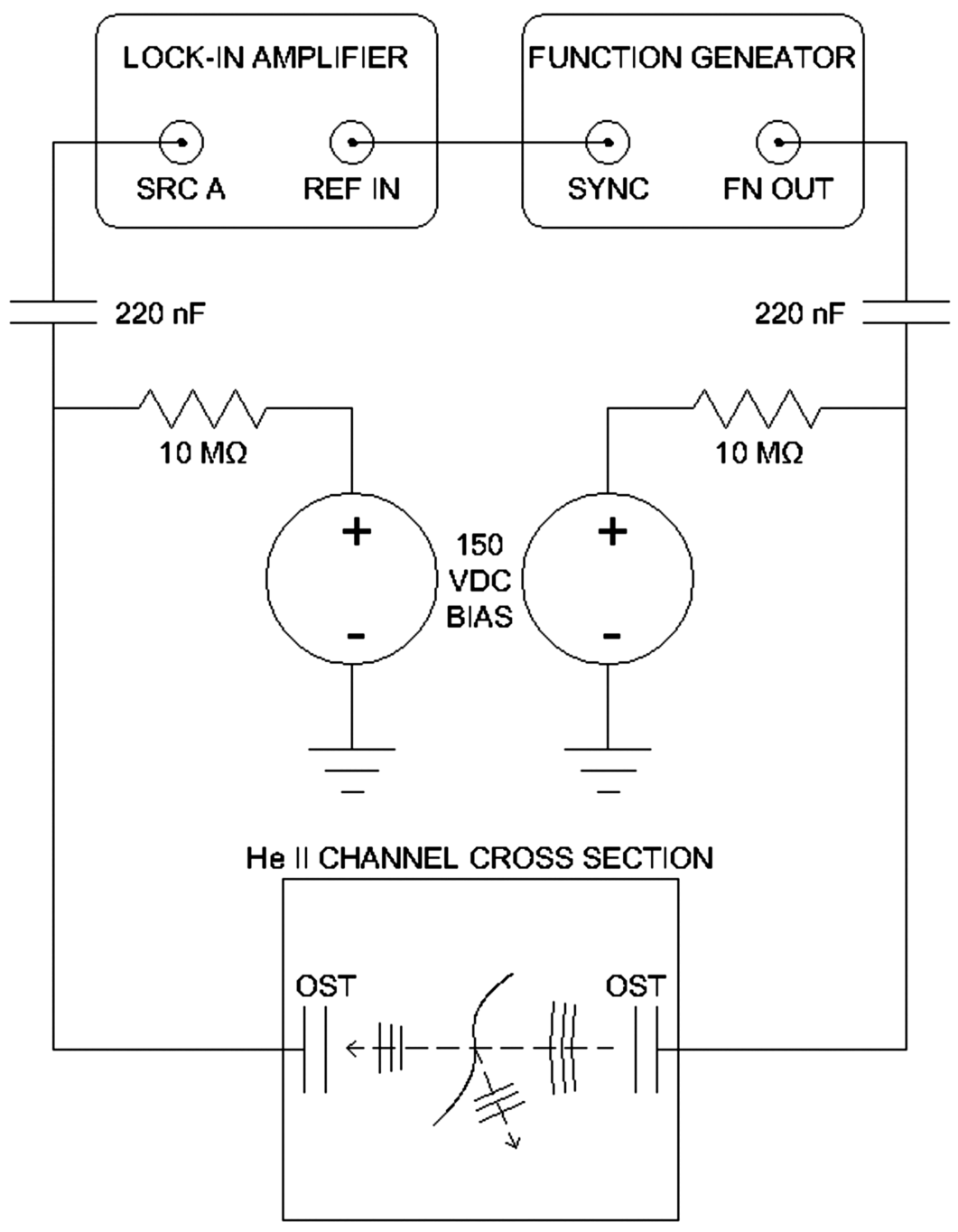

FIG. 8. Schematic of the second sound system. A 3.5 Vrms sinusoidal signal on top of a 150 VDC bias excites the transmitting OST, which produces a second sound wave in the $\mathrm{He}$ II filled channel. Second sound is scattered by quantized vortex lines; when the attenuated wave reaches the receiving OST, the induced AC signal is detected by a lock-in amplifier. 
sound wave impinging on a transducer causes the membrane to vibrate, inducing a measurable alternating current.

Measurement of vortex line density in a channel requires two second sound transducers placed across from each other on opposite sides of the channel (refer to Fig. 1). For this experiment, the second sound transducers have been designed for compatibility with the acrylic flow channel. They are fully selfcontained units that expose a flat sensing surface to the inside of the channel, and they have a built in Sub-Miniature version A (SMA) coaxial connector on the other side, as seen in Fig. 7. The porous membrane is stretched over a brass electrode and held in place by an aluminum retaining ring which makes contact with the gold coating. When assembled, the brass electrode connects with the center conductor of the coaxial signal cable. Indium wire provides continuity between the retaining ring and base flange, closing the circuit between the cable shield and the coated side of the membrane.

A schematic of the second sound measurement system is shown in Fig. 8. One transducer, driven at $3.5 \mathrm{Vrms}$ by a function generator, acts as the second sound transmitter, and the other picks up an attenuated signal at the same frequency, which is measured using a lock-in amplifier. Both transducers are biased with 150 VDC to flatten the membrane and improve the quality of the signal. $10 \mathrm{M} \Omega$ resistors prevent excessive current draw from the DC power supplies should a short develop across one or both membranes, and a pair of capacitors isolate the function generator and lock-in amplifier from the bias voltage.

\section{PERFORMANCE IN EARLY EXPERIMENTS}

Performance of the grid turbulence facility has been tested in preliminary experiments. Tracer particles have been successfully introduced into the channel, and several videos of their motion in the decaying turbulence after the grid movement have been recorded. The particle tracks have been extracted and, when a full data set is available, can be used for statistical analysis of the turbulent flow field. The second sound transducers can successfully generate and pick up second sound signals across a broad frequency spectrum, and a measurable attenuation is observed when vortices are present.

\section{A. Visualization experiment}

An example of the solidified deuterium particle tracks after the grid moves through the channel is shown in Fig. 9 (Multimedia view) (the original video is available online). In this particular case, the fluid temperature was $2.0 \mathrm{~K}$, the grid speed was $30 \mathrm{~cm} / \mathrm{s}$, and the grid mesh size was $3.75 \mathrm{~mm}$. Each track consists of, essentially, a list of spatial coordinates $(x, y)$ describing the particle position at a known time $t$. With an appropriately large number of tracks, a number of important statistical analyses can be performed.

As an example of one quantitative measurement that is possible with this velocity data, the probability density functions (PDFs) for measured particle velocity at $1 \mathrm{~s}$ intervals throughout the observed $7 \mathrm{~s}$ decay period have been calculated, a selection of which are shown in Fig. 10. These were obtained by extracting the particle velocity from track

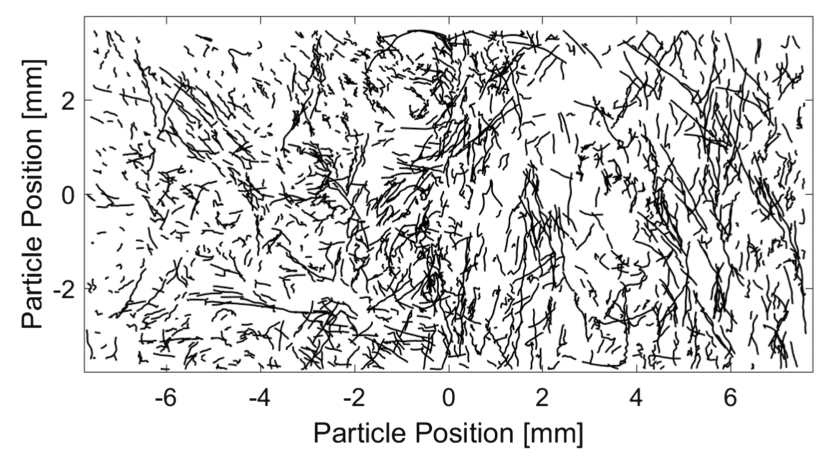

FIG. 9. Particle tracks in the first several seconds after pulling the $M=3.75 \mathrm{~mm}$ grid at $30 \mathrm{~cm} / \mathrm{s}$. The fluid temperature was $2.0 \mathrm{~K}$. The origin in the figure represents the geometric center of the channel. Playback of the online video is reduced to $30 \%$ of the original speed. Multimedia view: https://doi.org/10.1063/1.4997735.1

segments that appear $1 \pm 0.1 \mathrm{~s}$ after the grid passes the geometric center of the channel and $2 \pm 0.1 \mathrm{~s}$ after the grid passes the center. It can be clearly seen for both the $x$-direction
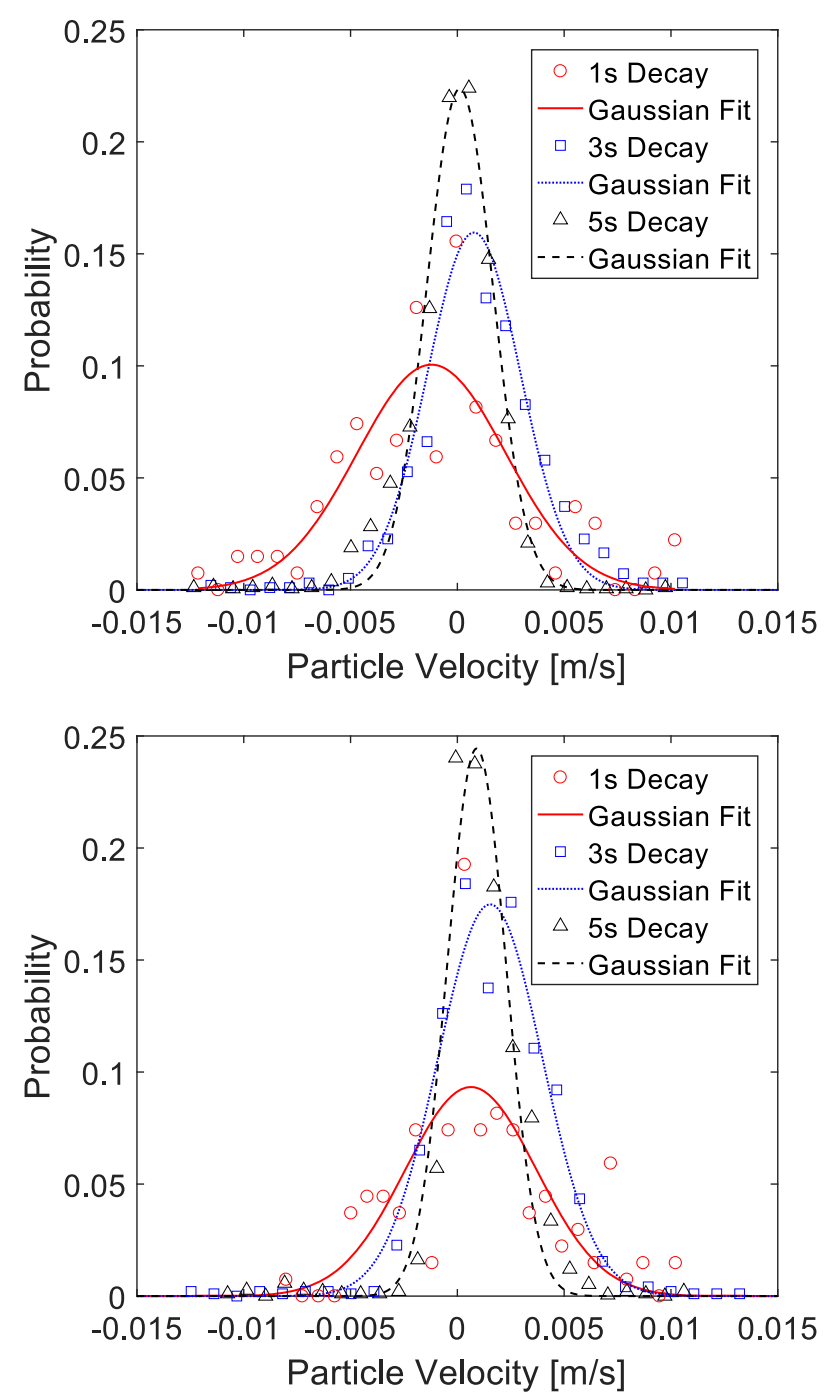

FIG. 10. (top) PDFs of the measured particle velocity along the $x$-direction (across the channel) at $2 \mathrm{~s}$ intervals for the first several seconds of the turbulence decay. (bottom) The same for the $y$-direction (along the channel axis). 


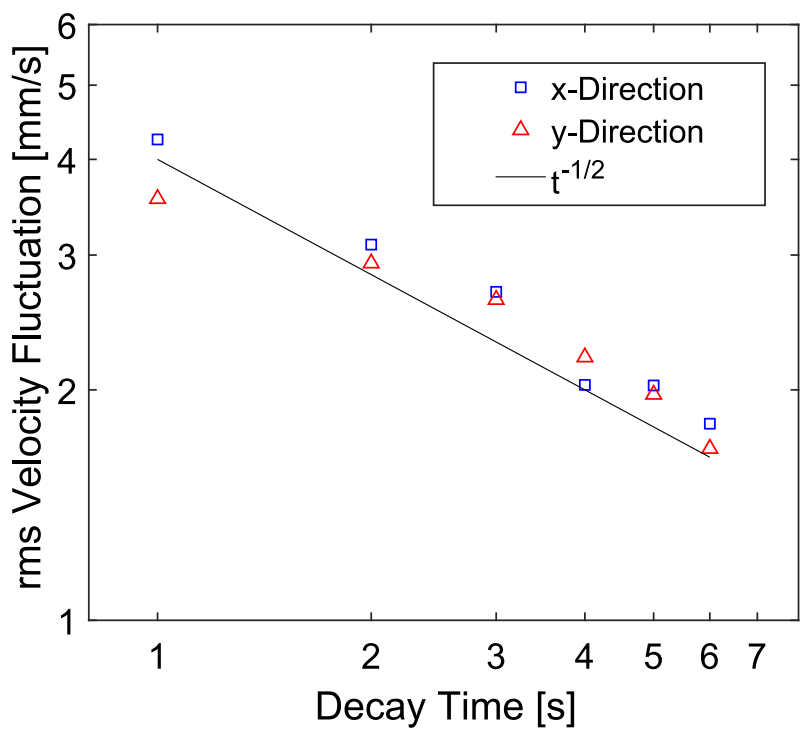

FIG. 11. rms fluctuation for the $x$-component and $y$-component of the measured particle velocity as a function of time after pulling the $M=3.75 \mathrm{~mm}$ grid at $30 \mathrm{~cm} / \mathrm{s}(T=2.0 \mathrm{~K})$.

(horizontal) and $y$-direction (vertical, along the axis of the channel) that the width of the PDFs decreases with increasing time, indicating that the turbulent kinetic energy density decreases with time, as expected for decaying turbulence.

To further quantify this observation, Fig. 11 shows the root mean square (rms) velocity fluctuation $u^{\prime}$ as a function of time, calculated by

$$
u^{\prime}=\sqrt{\frac{1}{N} \sum_{i=1}^{N}\left(u_{i}-\bar{u}\right)^{2}},
$$

where $N$ is the number of velocity samples $u$ measured in a $0.2 \mathrm{~s}$ time window centered at the indicated decay time. RMS velocity fluctuation is similar for both the $x$-component and $y$-component of velocity, a strong indicator of isotropy in the turbulent flow field, and the decay rate seems to be proportional to $t^{-1 / 2}$. Since the turbulent kinetic energy density $E$ is proportional to $u^{\prime 2}$, it follows that, in this case, $E \propto t^{-1}$. For homogeneous turbulence, the energy decay rate is proportional to the mean square vorticity according to

$$
\frac{d E}{d t}=-v\left\langle\omega^{2}\right\rangle
$$

where $\omega$ represents vorticity. ${ }^{17}$ This implies that $\omega \propto t^{-1}$, which can be verified by the second sound measurement discussed in Sec. III B.

\section{B. Second sound experiment}

The second sound frequency spectrum for the acrylic flow channel has been characterized for the case when $T=1.65 \mathrm{~K}$. This is accomplished by exciting one transducer with a sinusoidal signal of $3.5 \mathrm{Vrms}$ on top of a $150 \mathrm{VDC}$ bias, using the measurement system shown in Fig. 8, and recording the lockin amplifier response. The function generator steps through a wide range of frequencies, resulting in the spectrum of Fig. 12.

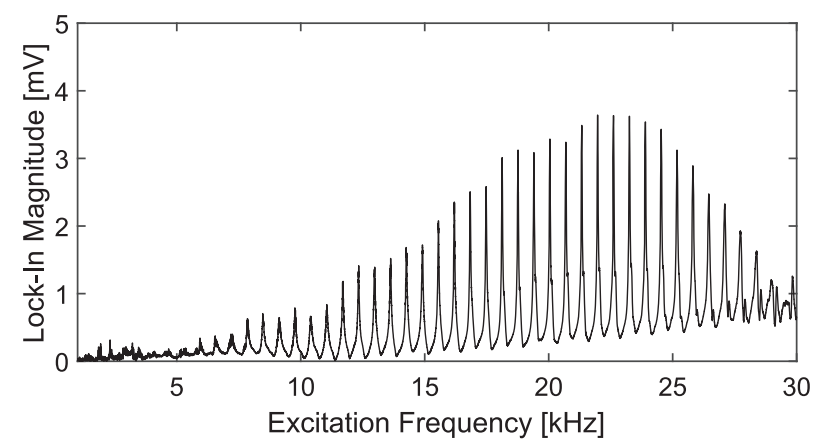

FIG. 12. Second sound frequency spectrum for the cast acrylic flow channel when filled with $\mathrm{He}$ II at $T=1.65 \mathrm{~K}$.

Each peak in the spectrum corresponds with a resonant frequency of the second sound wave in the channel. Taking into account the change in channel dimensions due to thermal contraction, the peaks should occur approximately every $643 \mathrm{~Hz}$ according to the relationship

$$
f=\frac{n c_{2}}{2 d},
$$

where $n$ represents the $n$th harmonic, $c_{2}$ represents the second sound velocity, and $d$ represents the width of the channel. On average, a resonant peak occurs in the measured spectrum every $642 \mathrm{~Hz}$.

Stepping the excitation frequency over a selected resonant peak while the fluid is stagnant establishes a baseline measurement for the peak amplitude, $a_{0}$, and the full width of the peak at half maximum height (FWHM), $\Delta_{0}$. When quantized vortices are present in the channel, the peak height is attenuated

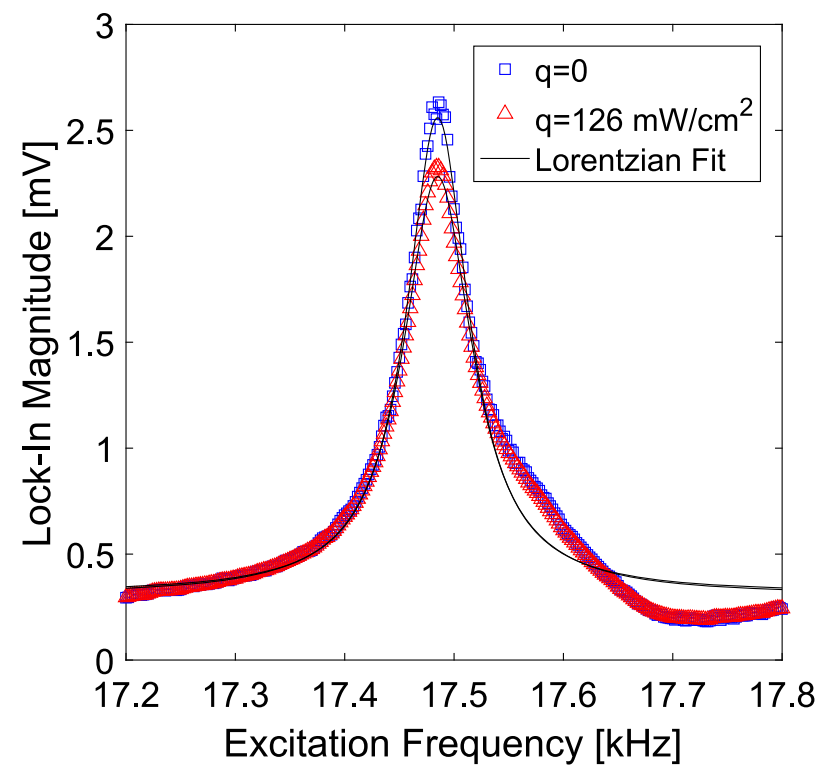

FIG. 13. Example of the attenuation of a second sound resonant peak due to the presence of quantized vortices. The peak shown by blue squares was acquired while the fluid was quiescent; a Lorentzian function fit to the data (black line) provides a baseline measurement of the peak height, $a_{0}$, and FWHM, $\Delta_{0}$. For purposes of this illustration, the heater was used to generate steady-state thermal counterflow turbulence during another acquisition of the same resonant peak. This height $a$ of this attenuated peak, shown by the red triangles, is clearly less than $a_{0}$. 


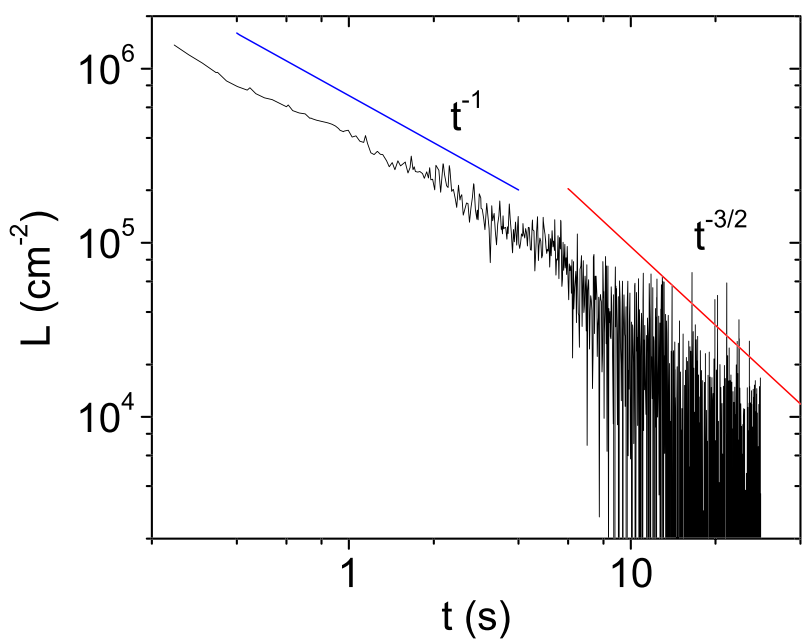

FIG. 14. Vortex line density as a function of time measured after pulling the $M=3.75 \mathrm{~mm}$ grid at $30 \mathrm{~cm} / \mathrm{s}(T=1.65 \mathrm{~K})$. Lines indicating some possible decay rates, $t^{-1}$ for the first several seconds and $t^{-3 / 2}$ for the later decay, are also shown.

proportionately to the average vortex line length per unit volume, $L$. This attenuation is illustrated in Fig. 13. After the grid movement, the time-dependent height of the resonance peak, $a(t)$, is measured continuously throughout the decay. Together with the measurements of the unattenuated peak, this can be used to calculate $L(t)$ by ${ }^{36}$

$$
L(t)=\frac{6 \pi \Delta_{0}}{B \kappa}\left(\frac{a_{0}}{a(t)}-1\right),
$$

where $B$ is an empirical constant. ${ }^{3}$

An example of the vortex line density measurement in decaying grid turbulence is shown in Fig. 14. In this case, the fluid temperature was $1.65 \mathrm{~K}$, the grid speed was $30 \mathrm{~cm} / \mathrm{s}$, and the grid mesh size was $3.75 \mathrm{~mm}$. The noise in the data is likely due to temperature fluctuation in the liquid helium bath; since the second sound velocity is a function of temperature, ${ }^{6}$ small temperature fluctuations cause the resonant frequency to shift during the measurement, introducing artificial attenuation. Carefully controlling the fluid temperature, as well as averaging together a large number of measurements, should reduce the noise. Nevertheless, an overall trend can be observed, and it appears that $L$ is proportional to $t^{-1}$ in the first several seconds of the decay. If the quantity $(\kappa L)^{2}$ is taken as mean square vorticity, ${ }^{8}$ so that $L \propto t^{-1}$ is analogous to $\omega \propto t^{-1}$ as predicted by (4), then the observations of the second sound measurement agree with those of the visualization experiment. This correlation essentially validates the purpose of this apparatus: to investigate towed grid turbulence in He II using two complementary experimental techniques.

\section{CONCLUSIONS AND FUTURE DIRECTION}

A novel apparatus that enables measurement of quasiclassical turbulent flow in the wake of a towed grid using two diagnostic methods, particle tracking velocimetry and second sound attenuation, has been described. Combination of the two measurement techniques in one facility is achieved through the design of a cast acrylic flow channel as well as grids, a pulling system, and customized instrumentation, all of which are carefully designed for compatibility with the intended measurements. Preliminary experiments show that quantitative information can be extracted by analyzing the motion of tracer particles in the turbulent flow field and that quantized vortex line density can be quantified by measuring second sound attenuation. Furthermore, the observations of both experimental methods strongly agree with each other.

In the future, detailed measurements can be performed to answer some open questions about quasiclassical turbulence in He II. For example, the proportionality constant in (4) is more accurately represented by an effective kinematic viscosity $v^{\prime}$, which describes the combined effect of the normal fluid viscosity and mutual friction, than by the kinematic viscosity of the normal fluid alone. ${ }^{37} v^{\prime}$ has been measured for grid turbulence in He II, but the calculation involved an assumption about the energy decay rate. ${ }^{37}$ More recently, a method for calculating $v^{\prime}$ in decaying thermal counterflow turbulence, which uses flow visualization together with second sound attenuation to avoid the need for assumptions, has been established; ${ }^{38}$ with the new apparatus, this more accurate method can be applied to calculate $v^{\prime}$ for decaying grid turbulence. The energy decay characteristics found using second sound attenuation for the superfluid and flow visualization for the bulk fluid can be compared to determine under what conditions the two fluid components are coupled together. Additional concerns, such as intermittency enhancement, can be addressed by more detailed statistical analysis, such as computation of higher order structure functions ${ }^{15}$ from the Lagrangian particle trajectories. The capability to perform such measurements while varying and carefully controlling fluid temperature, grid mesh size, and grid speed will allow systematic measurements of HIT in $\mathrm{He}$ II. These will provide better understanding of the nature of this fluid system and help to enable the use of cryogenic helium for practical fluid mechanics research.

\section{ACKNOWLEDGMENTS}

This work is supported by U.S. Department of Energy Grant No. DE-FG02-96ER40952. It was conducted at the National High Magnetic Field Laboratory, which is supported by NSF No. DMR-1157490 and the State of Florida. The authors wish to thank the NHMFL machine shop and MS\&T department for their guidance and assistance fabricating the apparatus.

${ }^{1}$ L. Skrbek, J. J. Niemela, and R. J. Donnelly, J. Phys.: Condens. Matter 11, 7761 (1999).

${ }^{2}$ K. R. Sreenivasan and R. J. Donnelly, Adv. Appl. Mech. 37, 239 (2001).

${ }^{3}$ R. J. Donnelly and C. F. Barenghi, J. Phys. Chem. Ref. Data 27, 1217 (1998).

${ }^{4}$ S. Fuzier, B. Baudouy, and S. W. Van Sciver, Cryogenics 41, 453 (2001).

${ }^{5}$ B. Saint-Michel, E. Herbert, J. Salort, C. Baudet, M. B. Mardion, P. Bonnay, M. Bourgoin, B. Castaing, L. Chevillard, F. Daviaud, P. Diribarne, B. Dubrulle, Y. Gagne, M. Gibert, A. Girard, B. Hébral, T. Lehner, and B. Rousset, Phys. Fluids 26, 125109 (2014).

${ }^{6}$ S. W. Van Sciver, Helium Cryogenics (Springer, 2012).

${ }^{7}$ W. F. Vinen, Proc. R. Soc. Lond. A 242, 493-515 (1957).

${ }^{8}$ S. R. Stalp, L. Skrbek, and R. J. Donnelly, Phys. Rev. Lett. 82, 4831 (1999).

${ }^{9}$ J. Maurer and P. Tabeling, Europhys. Lett. 43, 29 (1998).

${ }^{10}$ J. Salort, C. Baudet, B. Castaing, B. Chabaud, F. Daviaud, T. Didelot, P. Diribarne, B. Dubrulle, Y. Gagne, F. Gauthier, A. Girard, B. Hébral, B. Rousset, P. Thibault, and P.-E. Roche, Phys. Fluids 22, 125102 (2010). 
${ }^{11}$ S. Babuin, E. Varga, and L. Skrbek, J. Low Temp. Phys. 175, 324 (2014).

${ }^{12}$ W. F. Vinen, Phys. Rev. B 61, 1410 (2000).

${ }^{13}$ A. W. Baggaley, C. F. Barenghi, A. Shukurov, and Y. A. Sergeev, Europhys. Lett. 98, 26002 (2012).

${ }^{14}$ D. Kivotides, Europhys. Lett. 112, 36005 (2015).

${ }^{15}$ L. Boué, V. L'vov, A. Pomyalov, and I. Procaccia, Phys. Rev. Lett. 110, 014502 (2013).

${ }^{16}$ E. Rusaouen, B. Chabaud, J. Salort, and P.-E. Roche, Phys. Fluids 29, 105108 (2017).

${ }^{17}$ J. O. Hinze, Turbulence (McGraw-Hill, 1975).

${ }^{18}$ C. M. White, A. N. Karpetis, and K. R. Sreenivasan, J. Fluid Mech. 452, 189 (2002).

${ }^{19}$ R. Donnelly, A. Karpetis, J. Niemela, K. Sreenivasan, W. Vinen, and C. White, J. Low Temp. Phys. 126, 327 (2002).

${ }^{20}$ E. Fonda, K. R. Sreenivasan, and D. P. Lathrop, Rev. Sci. Instrum. 87, 025106 (2016).

${ }^{21}$ P. Švančara and M. La Mantia, J. Fluid Mech. 832, 578 (2017).

${ }^{22}$ SCIGRIP Americas, 600 Ellis Rd., Durham, NC 27703.

${ }^{23}$ Lake Shore Cryotronics, Inc., 575 McCorkle Blvd., Westerville, OH 43082.

${ }^{24}$ R. C. Dhuley and S. W. Van Sciver, Cryogenics 77, 49 (2016).
${ }^{25}$ H. J. S. Fernando and P. D. De Silva, Phys. Fluids A 5, 1849 (1993).

${ }^{26}$ R. E. Honey, R. Hershberger, R. J. Donnelly, and D. Bolster, Phys. Rev. E 89, 053016 (2014).

${ }^{27}$ BellowsTech, 1289 N U.S. Highway 1, Suite \#1, Ormond Beach, FL 32174.

${ }^{28}$ T. V. Chagovets and S. W. Van Sciver, Phys. Fluids 23, 107102 (2011)

${ }^{29}$ M. La Mantia, T. V. Chagovets, M. Rotter, and L. Skrbek, Rev. Sci. Instrum. 83, 055109 (2012).

${ }^{30}$ P. K. Kundu, I. M. Cohen, and D. R. Dowling, Fluid Mechanics (Elsevier, 2012).

${ }^{31}$ M. La Mantia and L. Skrbek, Phys. Rev. B 90, 014519 (2014).

${ }^{32}$ Changchun New Industries Optoelectronics Tech. Co., Ltd, No. 888 Jinhu Rd., Changchun, 130103 China.

${ }^{33}$ Integrated Design Tools, 1804 Miccosukee Commons Suite 208, Tallahassee, FL 32308.

${ }^{34}$ I. Sbalzarini and F. Koumoutsakos, J. Struct. Biol. 151, 182 (2005).

${ }^{35}$ R. A. Sherlock and D. O. Edwards, Rev. Sci. Instrum. 41, 1603 (1970).

${ }^{36}$ L. Skrbek and K. R. Sreenivasan, Phys. Fluids 24, 011301 (2012).

${ }^{37}$ S. R. Stalp, J. J. Niemela, W. F. Vinen, and R. J. Donnelly, Phys. Fluids 14, 1377 (2002).

${ }^{38}$ J. Gao, W. Guo, and W. F. Vinen, Phys. Rev. B 94, 094502 (2016). 